\title{
Development of Novel miRNA-based Vaccines and Antivirals against Enterovirus 71
}

\section{Running Title: miRNA-based Vaccines and Antivirals}

Pinn Tsin Isabel Yee and Chit Laa Poh*

Research Centre for Biomedical Sciences, Sunway University, Bandar Sunway, Kuala Lumpur, Selangor 47500, Malaysia

*Correspondence: pohcl@sunway.edu.my; Tel.: +603-7491-8622 (ext. 7338); Fax: +603-5635-8633

\begin{abstract}
The Hand, Foot and Mouth Disease (HFMD) is caused by Enterovirus 71 (EV-A71) and Coxsackieviruses. Common HFMD symptoms are high fever $\left(\geq 39^{\circ} \mathrm{C}\right)$, rashes, and ulcers but complications due to virulent EV-A71 may arise leading to cardiopulmonary failure and death. The lack of vaccines and antiviral drugs against EV-A71 highlights the urgency of developing preventive and treatment agents. Recent studies have reported the emergence of novel antiviral agents and vaccines that utilize microRNAs (miRNAs). They belong to a class of small (19-24 nt) non coding RNA molecules. As miRNAs play a major role in the host regulatory system, there is a huge opportunity for interplay between host miRNAs and EV-A71 expressions. A total of 42 out of 64 miRNAs were up-regulated in EV-A71-infected cells. There was consistent up-regulation of miR-1246 gene expression that targeted the DLG3 gene which contributes to neurological pathogenesis. In contrast, miR30a that targets calcium channels for membrane transportation was down-regulated. This leads to repression of EV-A71 replication. The impact of host miRNAs on immune activation, shutdown of host protein synthesis, apoptosis, signal transduction and viral replication are discussed. miRNAs have been used in the construction of live attenuated vaccines (LAV) such as the poliovirus LAV that has miRNA binding sites for let-7a or miR-124a. The miRNA-bearing vaccine will not replicate in neuronal cells carrying the corresponding miRNA but could still replicate in the gastrointestinal tract and hence remains to act as immunogens. As such, miRNAs are attractive candidates to be developed as vaccines and antivirals.
\end{abstract}

Keywords: MicroRNA, Enterovirus 71, genes, antivirals, vaccines, transcription, translation. 


\section{INTRODUCTION}

The hand, foot, and mouth disease (HFMD) is generally manifested as a mild illness but fatal neurological complications leading to cerebellar encephalitis, acute flaccid paralysis and aseptic meningitis have been observed in infants and young children below 6 years of age (1). The EV-A71 virus was first isolated as the etiological agent of HFMD from a young child in California, United States in 1969 (2). The virus is a member of the human Enterovirus species A belonging to the Picornaviridae family (3). HFMD outbreaks caused by EVA71 infection have been occurring periodically throughout the world. Since 1997, countries such as Taiwan, Japan, Malaysia, Singapore and Vietnam had experienced cyclical epidemics which occurred every 2 or 3 years (4). HFMD-caused by enteroviruses such as Enterovirus 71 (EV-A71), Coxsackie type A16 (CV-A16), CV-A10, CV-A6 and other enteroviruses causing HFMD have led to over 7 million infections, including 2457 fatalities in China from 2008 to 2012 (5).

With rising concern about the virulence of EV-A71, there is an urgent need for a vaccine against EVA71 to be produced that is approved by the United States Food and Drug Administration (FDA) or European Medicines Agency (EMEA). Besides traditional approaches such as the EV-A71 inactivated vaccine which is at the forefront of development, recent studies have reported the emergence of novel vaccines that utilize microRNAs (miRNAs). Although the inactivated vaccine (IV) is the safest vaccine as there will be no reversion to wild type, efficacy was only at $80 \%$ against serious HFMD cases (6). Although the IV would be able to reduce the number of mild HFMD cases, there would be $20 \%$ of the population that remain susceptible to severe HFMD infection. Additionally, the IV only induces humoral immunity and lacks cellular immunity. With an IV, there is no viral replication and hence there is lower antigen content and less prolonged antigen persistence (7). As a result, inactivated vaccines elicit weaker and shorter antibody responses and there is no long-term immune memory. Hence, immunogenicity is not long-lasting and requires multiple boosters.

The live attenuated vaccine (LAV) is cheaper to produce, induces excellent immunogenicity and confers live-long immunity. LAV is preferred over IVs as it can elicit both humoral and cellular immunity. There is an increasing body of research which indicates that cellular and not humoral immunity determines the clinical outcome of EV-A71 infections. In the most severe cases with pulmonary edema, blood samples showed lower levels of cellular cytokines and interferons (TNF- $\alpha$, IL-1 $\beta$ and IL-6), while there was no difference in the level of neutralizing antibody (NtAb) titers between mild, severe and fatal cases (8). Many diseases such as measles, mumps, influenza, rotavirus and rubella have safe and effective LAVs, testifying to the success and potential of LAVs. With advances in molecular biology, novel approaches to LAV can be further developed which relied on 
concepts such as altered replication fidelity, codon deoptimization and microRNA-controlled LAVs. Arita et al. (2007) attempted to design a LAV against the EV-A71 BrCr strain (S1-3') by incorporating 3 mutations in the 5'NTR (nt. 485, 486, 474), 2 mutations in the RdRP (Tyr-73, Cys-363) and a single site mutation in the 3'-NTR (nt. 7409). Although the strain had reduced pathogenicity, cynomolgous monkeys inoculated with the S1-3' strain still showed mild neurological manifestations and the virus was still isolated from the spinal cord (9). Hence, the plan to use this strain as a LAV was discontinued.

\section{DEVELOPMENT OF MIRNA-BASED VACCINES}

miRNAs are 19-24 nucleotides long, non-coding RNAs that can prevent translation of messenger RNA (mRNA). The first miRNA, lin-4, was isolated from an unsegmented roundworm, Caenorhabditis elegans (C. elegans) in 1993. After several years the second miRNA, let-7 was also discovered in C. elegans (10). Since its first discovery, thousands of new miRNAs have been identified from a diverse range of organisms, including humans (11). A number of viruses, predominantly from the large dsDNA virus family, have been found to express their own miRNA, for example, herpesviruses and polyomaviruses (12). As miRNAs present in the human genome are known to interact with viruses, recent advances in the molecular interplay between EV-A71 and miRNAs will be reviewed.

The primary function of miRNA is RNA silencing and post-transcriptional regulation of gene expression. miRNA is able to repress translation or induce mRNA degradation, depending on its sequence homology with the targeted mRNA (11). Complete complementarity between miRNA and target mRNA would lead to cleavage and degradation while mismatches would lead to suppression of translation. MicroRNAs are genomically encoded and transcribed as a long precursor pri-miRNA. Drosha, a nuclear ribonuclease (RNAse) and DGCR8 (DiGeorge Syndrome Critical Region) would then process the pri-miRNA to $\sim 60$ nucleotide (nt) hairpin intermediates (premiRNA) which are transported to the cytoplasm (Fig. 1). The hairpin intermediate would thereby be trimmed by a second RNAse II, Dicer to 22 nt. prior to being loaded onto the RNA-induced silencing complex (RISC). This would then lead to either mRNA degradation or translational inhibition (13).

The biogenesis process is found in almost all organisms with the exception of RNA viruses. There is little evidence for miRNA being produced by RNA viruses in contrast to DNA viruses (14). One of the reasons is that the miRNA biogenesis machinery via Drosha is located in the nucleus, whereas the genomes of cytoplasmic viruses are located the cytoplasm and are physically separated from Drosha and its cofactors. The processing of miRNAs from RNA viral genomes in the cytoplasm would result in the cleavage of the genome during excision of the miRNA, rendering it useless as a template for translation or replication. 


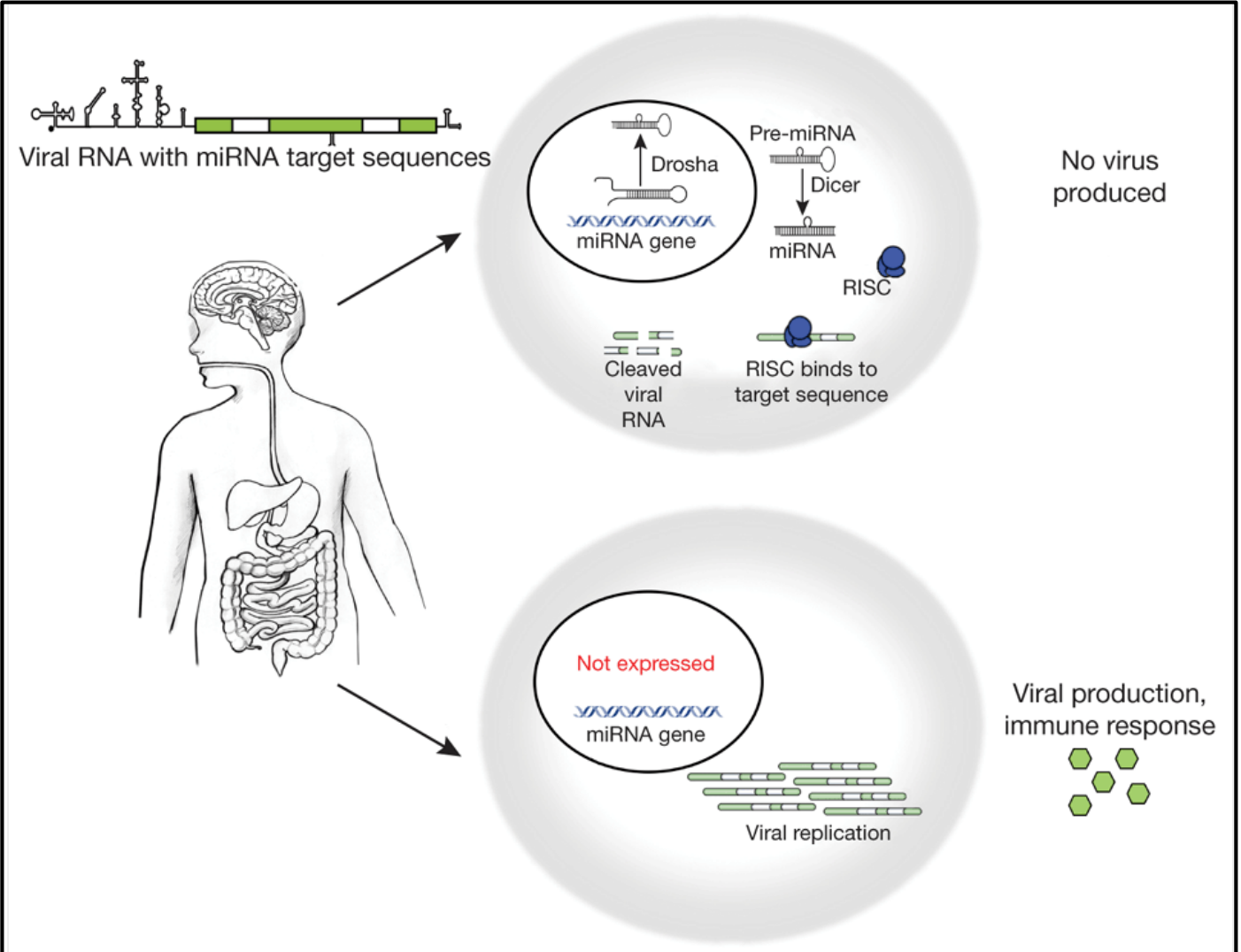

Fig. (1). Biogenesis of miRNA and miRNA based vaccine strategy.

Silencing of miRNA transcripts can be achieved in a tissue-specific manner through insertion of a miRNA target sequence into a virus vector. In neuronal cells that express the miRNA, the viral RNA is cleaved and there is no virus produced. In intestinal cells which do not express the miRNA, viral replication is restricted and an immune response is elicited without the associated risk of disease. Adapted by permission from Macmillan Publishers Ltd: [Nature Biotechnology] (13), copyright (2010). 
The human genome has more than 1000 different miRNAs that are tissue specific, which can function as post-transcriptional regulators, capable of repressing hundreds of genes and regulating many cellular processes $(15,16)$. miRNAs are found to regulate functions such as cell proliferation, differentiation, homeostasis, immune activation and apoptosis $(12,17-20)$. miRNAs have tissue-specific signatures and their expression profiles are unique for every tissue. It was discovered that the small intestines of mice express higher amounts of miR-143 and miR-194 as compared to other tissues (21). Host-encoded miRNAs have also been found to participate in complex host-virus interactions (22). New technologies are emerging on the utilization of miRNA target sites for the inhibition of endogenous miRNA (23). This can be carried out through the insertion of a miRNA target sequence into a genome sequence in a vector thereby, silencing a particular miRNA transcript.

Brown et al. (2006) incorporated miR-142-3p binding sites into their lentiviral vector and discovered that expression from hepatocyte specific promoters was suppressed in hematopoietic lineages. This expression profile was not repressed in non-hematopoietic lineages in immunocompetent mice (24). This meant that gene expression of the integrated miRNA target sequence was controlled by cellular miRNAs in target tissues. In addition, Kelly and colleagues (2008) demonstrated that insertion of a muscle-specific miRNA target sites (miR133 and miR-206) into the 3'-NTR of CVA-21 repressed expression of lethal myositis in tumor bearing mice. It was observed that there was lethal myotropism in viruses that had a disrupted miRNA target sequence (25). A later study (2010), reported that the picornaviral life cycle could be disrupted by miRNAs even at very late stages after infection, albeit at very high levels of viral copy number. In fact, the life cycle could be interrupted at multiple levels, including catalytic degradation of the viral genome, suppression of uncapped IRES-dependent mRNA translation, and interference with viral encapsidation (26). The virus would not be able to replicate in cells expressing the corresponding miRNA and would thereby display an attenuated phenotype (27).

In another study, inclusion of hepatocyte-specific miRNA target sequences for miR-122 at the 3'-NTR of the adenovirus genome showed an 80-fold decrease in hepatic expression of adenovirus after intravenous delivery to mice (28). Perez et al. (2009) demonstrated that by incorporation of miRNA target sites into the open reading frame of the Influenza A viruses, they could produce attenuated H1N1 and H5N1 viruses. These attenuated viruses led to a 2-log reduction of mortality and a diverse antibody response in mice (29). Lee and coworkers (2010) inserted the hepatocyte specific miR-122 into the 3'-NTR of the dengue virus and found that liver infection was effectively suppressed (30). These studies corroborate intensive research that cellular miRNAs can effectively inhibit pathogenic viral replication and provide an additional layer of biosafety. 
An ideal attenuated virus vaccine should be one that does not replicate in tissues that it can cause disease and yet at the same time, replicate actively in other tissues to elicit a complete and long-lasting immune reaction. For example, the Sabin poliovirus replicates normally in many tissues but does not replicate in neuronal cells where it is able to cause lytic infection. Using the miRNA-based approach, Barnes and coworkers (2008) inserted miRNA binding sites for let-7a or miR-124a into the poliovirus genome and showed that there was restricted viral replication in the nervous system of a murine model. Although the degree of attenuation of neurovirulence exceeded 5 orders of magnitude, these engineered miRNA-bearing viruses were still able to replicate in nonneuronal tissues and elicit high neutralizing antibody titers $\left(10^{3}-10^{5}\right)$ after a single intraperitoneal injection in mice (26). These studies were consistent with previous reports that mice vaccinated with miRNA vaccines were able to elicit comparable neutralizing antibody titers as the Sabin type 1 poliovirus vaccine strain and were protected from the wild type virus (31). This meant that these vaccine candidates could stimulate a strong protective immunity without the adverse risk of causing paralysis.

Nevertheless, this strategy has its drawbacks as it was discovered that the let-7a bearing poliovirus did not replicate well in most tissues. This would then elicit a weaker immune response and the vaccine strain may have low immunogenic properties. In addition, although the miR-124a virus could not replicate in neuronal tissue, it was able to grow extensively in other tissues. However, this allows the virus to accumulate escape mutations that could prevent degradation of the viral genome. This also increases the potential for the miR-124a bearing virus to revert to the wild type poliovirus. It was also reported that several mice had low titers of mutated miR124a virus in their spinal cords. Therefore, it is essential to find ways to control the mutability of miRNA-based vaccines. One such method would be to incorporate a combination of miRNA target sequences into the viral genome to minimize escape from replication-silencing activity, increase cell selectivity and eliminate potential for cytopathic side effects (26). In addition, high fidelity imposed by a mutation can be engineered into viruses containing multiple miRNA target sequences. This would also serve to increase the genetic stability of the engineered virus and minimize the risk of reversion of the miRNA-bearing mutant to the wild type. These strategies can pave the way for the development of safe and effective vaccines to target viral diseases that do not yet have vaccines for prevention.

\section{2. miRNA ANTIVIRAL AGENTS}

miRNAs can either stimulate or suppress viral gene expression. A well-known example of a miRNA that is highly abundant and expressed in the liver is miR-122. This miRNA encourages hepatitis C viral (HCV) replication by promoting viral RNA stability. This was caused by the binding of host miR-122 to two closely 
spaced target sites (S1 and S2) in association with protein argonaute-2 (Ago2) in the 5'-NTR of the HCV genome, thereby forming an oligomeric miR-122-HCV complex. This binding masks the 5 ' terminal sequences from Xrn 1 degradation and promotes viral RNA stability. The miR-122 also enhances the association of small-unit ribosomes with viral RNA at the early initiation stage and stimulates translation of the HCV genome (32-33).

It is a well-known fact that viruses are recognized by host cell receptors and the host would elicit the endosomal toll-like receptor (TLR) signaling to produce Type I Interferons (IFNs) which serve as the earliest host response against viral infections (34-37). The IFNs would then activate downstream interferon-stimulating genes, T cell proliferation and IFN- $\lambda$ production (38). Studies have reported that Type 1 IFN production is inhibited by EV-A71 infection $(39,40)$. Interestingly, using miRNA prediction programs and 3'-UTR targeting site assays, Li et al (2012) found that the miR-548 family increased EV-A71 and vesicular stomatitis virus (VSV) infection by binding to the 3 '-UTR of IFN- $\lambda 1$, thereby down regulating expression of IFN- $\lambda 1$. When IFN- $\lambda 1$ inhibitors were added, this significantly suppressed the replication of EV-A71 through the up regulation of IFN- $\lambda 1$. Their studies suggested that miR-548 regulates host antiviral response via direct targeting of miR-548 inhibitors (41).

Lui and co-workers (2014) also demonstrated that EV-A71 utilized host miRNAs such as miR-548 to enhance the cellular microenvironment for viral replication. The miR-548 is involved in interferon (IFN) signaling pathway during viral infection. EV-A71 altered host miRNAs such as miR-548 to manipulate host antiviral responses. Up-regulation of miR-548 was found to down regulate various antiviral signaling pathways such as Toll-like receptor, Nod-like receptor, RIG-1-like receptor and Type 1 interferon signaling. They were able to investigate the role of an essential cofactor for miRNA biogenesis, a double-stranded-RNA-binding protein (DGCR8) that processes pri-miRNA to functional miRNAs. This was corroborated when the knock-down of DGCR8 gene resulted in the inability of EV-A71 to replicate in its host through the disruption of miRNA biogenesis. This is shown in the decrease in RNA expression levels of EV-A71 in miRNA-depleted RKO, HT29 and RD cells. EV-A71 was unable to establish infection in miRNA-depleted cells and this would mean that EVA71 uses host microRNAs to mediate the immune system such that it enhances viral survival during infection (42).

Ho et al. (2011) showed that EV-A71 up-regulated miR-141 expression which contributed to the shutoff of host protein translation by targeting the translation initiation factor eIF4E. Knockdown of miR-141 reduced viral propagation and silencing of eIF4E completely reversed the inhibitory effect of anti-miRNA oligonucleotides against miR-141 on viral propagation. They also discovered that a transcription factor, early growth response protein 1 (EGR1) was partly responsible for miR-141 induction in response to Enterovirus infection (43). In 
contrast, Zheng and co-workers (2013) discovered that EV-A71 infection up-regulates expression of cellular miR296-5p. The copy number of miR-296-5p in RD cells increased from 400 to 970 within 12 hours post infection (hpi), while in SK-N-SH cells, it ranged from 2,500 to 9,000 during EV-A71 infection. In addition, they also showed that the up-regulation of hsa-miR-296-5p inhibited EV-A71 infection as proven by the reduction in viral titer and EV-A71 RNA levels for hsa-miR-296-5p infected cells. Consistent with previous studies, VP1 protein levels were also found to decrease in hsa-miR-296-5p-transfected cells at 6 and 12 hpi based on VP1 immunoblotting. In contrast, inhibition of miR-296-5p facilitated EV-A71 pathogenesis as shown by significant increases in viral titer and RNA copy number after 8 hpi when cells were transfected with miR-296-5p inhibitors. Western blots indicated that the amounts of VP1 protein increased in miR-296-5p inhibitor-transfected cells at 6 hpi and 12 hpi. It was postulated that overexpression of miR-296-5p suppressed EV-A71 replication by targeting two potential miR-296-5p targets (nt. 2115 to 2135 and nt. 2896 to 2920) located in the EV-A71 genome of the $\mathrm{BrCr}$ prototype strain. They also demonstrated the introduction of synonymous mutations into the viral genome impaired the viral inhibitory effects of miR-296-5p (44). This shows that miR-296-5p suppresses EV-A71 gene expression by targeting the viral genome.

Cellular miRNA can also suppress viral infection as shown by Wen et al (2013) that expression of miR23b suppressed EV-A71 replication through the down-regulation of Viral Protein 1 (VP1). Their results showed that up-regulation of miR-23b targeted the 3'-NTR of EV-A71 genome and hence, down-regulated VP1 protein. Western blot analysis and viral titers for miR-23b mimics in EV-A71 infected cells were significantly lower as compared to cells infected with EV-A71 only (45). Cui and colleagues (2010) performed a deep sequencing approach to identify miRNAs involved in host response to EV-A71 infection. They demonstrated that 42 out of 64 miRNAs were up-regulated in EV-A71-infected human epidermoid carcinoma cells (Hep2). They discovered that there was consistent up-regulation of miR-1246 and miR-1237 gene expressions. In addition, miR-619, miR1268, miR-1273, miR-1290, miR-1178 and miR-1302-8 were up-regulated in EV-A71 infected cells. In contrast, miR-30a and miR-222 were down-regulated (22). Table 1 summarizes the miRNAs that are involved in EV-A71 infection.

In addition, EV-A71 has been found to up-regulate the expression of let-7b in neuronal SH-SY5Y cells. Let-7b induces apoptosis of EV-A71-infected cells by repressing cyclin D1 (CCND1), a vital element in apoptosis. Suppression of CCND1 leads to higher rate of apoptosis in infected cells. Hence, inhibition of let-7b by 2'-OMethyl-RNA restored CCND1 expression, prevented occurrence of apoptosis and reduced replication of EV-A71 in SH-SY5Y cells (46). In a separate study, miR-1246 which was up-regulated in response to EV-A71 infection 
was found to suppress expression of the disc-large homolog 3 (DLG3), which led to impairment of the neurological system. Reduction of DLG3 expression by miR-1246 could lead to meningitis and encephalitis which are symptomatic of severe EV-A71 infection. Hence, miR-1246 is postulated to play a role in neurological pathogenesis by the regulation of DLG3 expression (47).

The miRNAs can be silenced by using modified anti-miRNA oligonucleotides (AMOs), also known as "antagomirs" (48-52). They contain oligonucleotides complementary to their target sequence and can form a duplex with the target miRNA, thereby inhibiting the expression of the miRNA. Such an approach was first developed by Boutla and co-workers (54) whereby different antagomirs successfully inhibited miRNA in cell culture $(50-52,54)$, flies $(51,53,55)$ and mice (56-58) This could be attributed to the design of the oligonucleotides that had locked nucleic acids (LNA), with nuclease resistance and cholesteryl functionality at the 3' end for enhanced cellular uptake $(59,60)$. The key requirements for antagomiRs are that they must be cell permeable, not rapidly excreted and stable in vivo (61). However, they all require transfection agents which maybe cytotoxic in humans, to mediate entry into cells. Hence, to overcome this problem, the chemically modified oligonucleotides should be conjugated to cholesterol to facilitate cellular uptake. Another example of chemical modification includes the 2'-O-methyl-group (OMe)-modified oligonucleotides and locked nucleic acid (LNA)modified oligonucleotides, in which the 2'-O-oxygen is bridged to the 4'-position through a methylene linker to form a rigid bicycle, locked into a C3'-endo (RNA) sugar conformation (62).

Nevertheless, this strategy is not without controversy as there has been some concern about frequent viral mutations, particularly for RNA viruses that may impede the usage of such therapeutic miRNAs. Viruses have been known to mutate to escape suppression by cellular miRNAs. However, miRNAs work by imperfect complementarity and hence, this would pose an advantage to prevent mutational escape (63). There have been some suggestions on the degree of mutations that are tolerable for miRNA binding. Grimson et al. (2007) demonstrated features that are vital for posttranscriptional repression and that would be the degree of AU-richness around target sites, target site location relative to the stop codon and preferential base pairing to nt $13-16$ of the miRNA. Importantly, for target recognition would be the $\sim 7$-nt sites that match the binding region of the miRNA (64).

The usage of antagomirs has successfully been carried out against EV-A71 and HCV. Better antivirals can be produced with a greater understanding of cell signaling pathways and the effect of antagomirs in viral inhibition. It is known that EV-A71 inhibits induction of downstream IFN-stimulated genes (65). It is also unfortunate for the failure to use IFN for treatment of EV-A71 infection. In a typical virus-host interaction, the virus would be 
recognized by host pattern-recognition receptors and activate endosomal toll-like receptor (TLR) signaling to produce type I IFNs. The resulting IFNs would then promote the proliferation of memory T cells, stimulate IFN $\gamma$ production and activate natural killer cells. The two key elements in TLR signaling and IFN production are the Interleukin-1 receptor-associated kinase 1 (IRAK1) and TNF receptor-associated factor 6 (TRAF6). Ho and colleagues (2014) showed that EV-A71 infection suppressed TRAF6 and IRAK1 by the up-regulation of miR146a (Fig. 2). EV-A71 infected cells showed suppressed protein expression of IRAK1 and TRAF6 most significantly at 8 and $12 \mathrm{hpi}$. It was also demonstrated that induction of miR-146a and suppression of IRAK1 and TRAF6 were dose dependent in Rhabdomyosarcoma (RD) and mouse embryonic fibroblast (MEF) cells. Conversely, when the protein expression levels of IRAK1 and TRAF6 were restored remarkably, viral replication was inhibited and virus-infected neonatal mice survived EV-A71 lethal infection using miR-146a antagomirs. Intraperitoneal injection of the antagomiR-146a $(1.2 \mathrm{mg} \mathrm{kg}-1)$ at $1 \mathrm{~h}$ before virus infection significantly improved the survival rates (56-80\%) as compared with the PBS and antagomiR negative control groups (22 and 25\%, respectively) (66). 
Table 1. miRNAs up-regulation and down-regulation in EV-A71 infection.

\begin{tabular}{|c|c|c|c|}
\hline miRNAs & $\begin{array}{l}\text { Up- } \\
\text { regulation/ } \\
\text { Down- } \\
\text { regulation }^{\text {a }}\end{array}$ & Target Genes & Functions \\
\hline $\operatorname{miR}-1246$ & + & DLG3 & $\begin{array}{l}\text { Contribution to neurological } \\
\text { pathogenesis }\end{array}$ \\
\hline miR-141 & + & eIF4E & Enhancement of EV-A71 propagation \\
\hline $\operatorname{miR}-296-5 p$ & + & $\begin{array}{l}\text { EV-A71 VP3 and VP1-coding } \\
\text { region }\end{array}$ & Repression of viral replication \\
\hline $\operatorname{miR}-370$ & + & SOS1 and GADD $45 \beta$ & $\begin{array}{l}\text { Enhancement of EV-A71-induced cell } \\
\text { apoptosis }\end{array}$ \\
\hline $\operatorname{miR}-27 a$ & - & EGFR & Repression of viral replication \\
\hline miR-146a & + & IRAK1 and TRAF6 & Enhancement of viral pathogenesis \\
\hline $\operatorname{miR}-548$ & + & IFN- $\lambda 1$ & $\begin{array}{l}\text { Enhancement of replication of EV- } \\
\text { A71 and VSV }\end{array}$ \\
\hline $\begin{array}{l}\operatorname{miR}-636, \operatorname{miR}-1226 \\
\text { miR-421, miR-140 }\end{array}$ & + & Ribosomal protein S6 kinase & $\begin{array}{l}\text { Up-regulates kinases and } \\
\text { phosphatases }\end{array}$ \\
\hline $\operatorname{miR}-1178, \operatorname{miR}-140$ & + & $\begin{array}{l}\text { Transforming growth factor, } \\
\text { alpha }\end{array}$ & $\begin{array}{l}\text { Up-regulates cell signaling, } \\
\text { extracellular communication }\end{array}$ \\
\hline $\begin{array}{l}\text { miR-1254, miR-320c } \\
\text { miR-627, miR-21 }\end{array}$ & + & Cyclin-dependent kinase 6 & Up-regulates cell cycles \\
\hline $\begin{array}{l}\text { miR-1180, miR-582 } \\
\text { miR-545, miR-30a }\end{array}$ & - & $\begin{array}{l}\text { Calcium channel, voltage } \\
\text { dependent, L type, alpha IC } \\
\text { subunit }\end{array}$ & $\begin{array}{l}\text { Down-regulates membrane } \\
\text { transporters }\end{array}$ \\
\hline miR-26a, miR-27 & - & BCL2-antagonist/killer 1 & Down-regulates apoptosis \\
\hline miR-26a, miR-545 & - & $\begin{array}{l}\text { Muscle RAS oncogene } \\
\text { homolog }\end{array}$ & Down-regulates oncogenes \\
\hline
\end{tabular}

${ }^{\mathrm{a}}(+)$ denotes up-regulation of the miRNA upon EV-A71 infection. (-) denotes down-regulation of the miRNA upon EV-A71 infection. 
Many pharmaceutical companies are interested in initiating clinical trials to produce safe and effective antivirals using miRNA biology. For example, Mirna Therapeutics, Inc. (USA) is currently conducting a multicenter, dose-escalation study for clinical trial phase I, to investigate the safety, pharmacokinetics and pharmacodynamics of the miR-34 mimic, MRX34, in patients with primary or advanced liver cancer. The primary objectives of the clinical trial are to establish the maximum tolerated dose and the recommended Phase 2 dose for future clinical trials (67). The antagomirs against miR-122 to target HCV have also been tested in chimpanzees and results have been very promising. This anti-miR-122 molecule is the first miRNA-targeting treatment to enter human clinical trials in the fight against HCV (Miravirsen, Santaris Pharma A/S) (68). However, there is a possibility that this anti-miR-122 may interfere with hepatocyte metabolism. Hence, there is a need to search for other liver-specific miRNAs that may play a vital role in HCV-host interactions.

An interesting and alternative method to interfere with miRNA function would be the use of octaguanidium-conjugated vivo-morpholino oligonucleotides (vivo-MOs). They are single stranded (ss) DNA antisense agents that prevent translation of viral RNA by steric blocking of complementary RNA sequences and can even inhibit pri-mRNA or pre-miRNA. Tan et al. (2014) discovered that vivo-MOs targeting the IRES region of the EV-A71 genome showed significant viral plaque reductions and decreased viral RNA copy number. The vivo-MO-2 demonstrated antiviral activities against PV and CV-A16. However, an EV-A71 mutant that was resistant to vivo-MO-1 arose after eight passages in the presence of increasing concentrations of vivo-MO-1. The mutant contained a single substitution from Thymine to Cytosine at nt. 533 (69). Hence, additional studies need to be performed to examine the resistance of potential antivirals against EV-A71. In another study, Kloosterman et al. (2007) used vivo-MOs targeting miR-375 to cause defects in the morphology of pancreatic islet cells of zebrafish. The vivo-MOs could block miRNA maturation at the Drosha or Dicer cleavage steps, and thereby inhibit the activity of the mature miRNA (70). The usage of vivo-MOs holds great potential in unravelling miRNA function in the development of human and mammalian genes. Many research teams are also very interested to sequence the entire "miRNome" and understand the role played by different miRNAs in the pathogenesis of viral diseases. Indeed, anti-miRNAs have a promising potential to be developed into novel anti-viral agents to treat patients infected with EV-A71. 


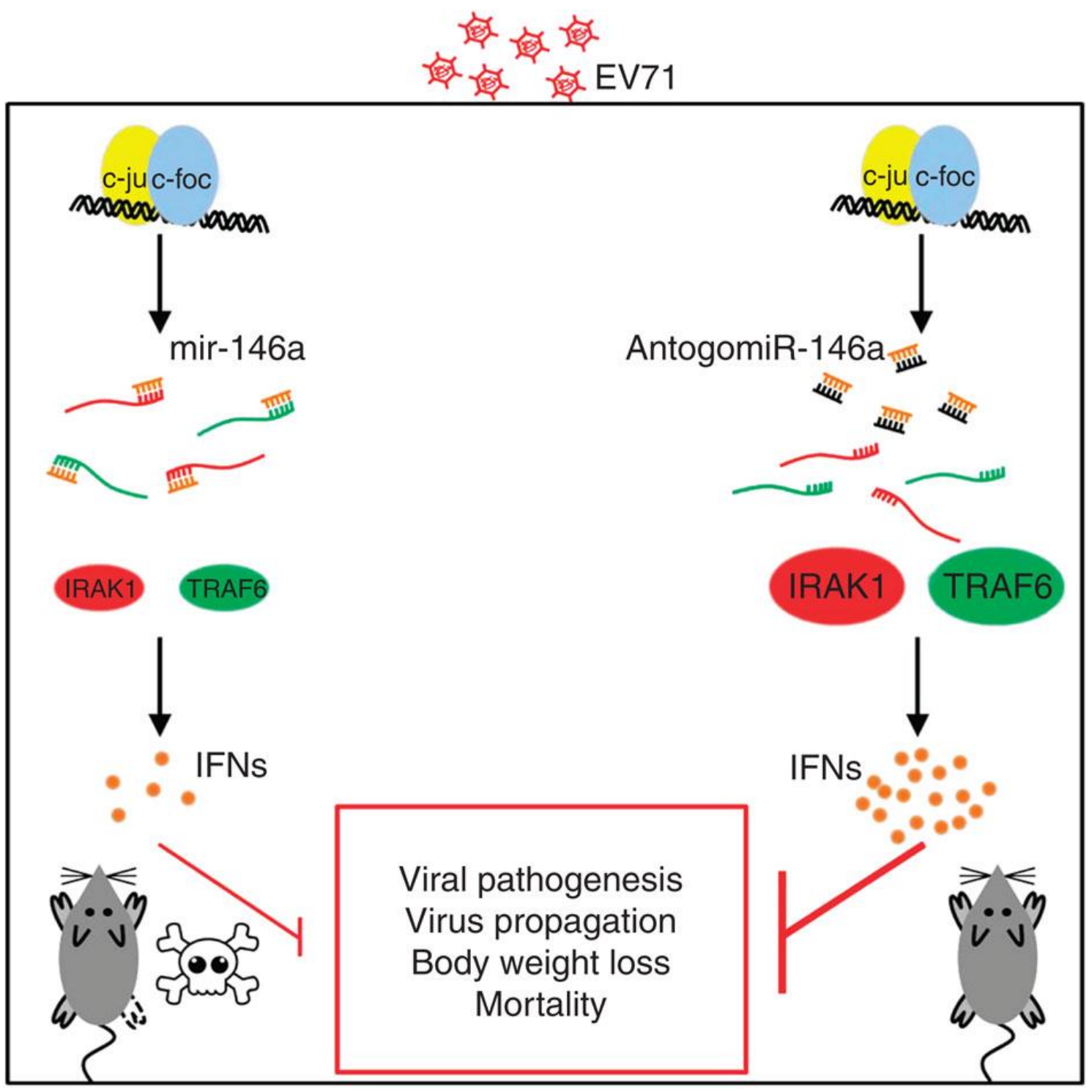

Fig. (2). EV-A71 infection up-regulates miR-146a, which suppresses expression of IRAK1 and TRAF6.

This inhibits production of type I IFN and virus escapes host immune attacks. Neutralization of virus-induced miR-146a by antagomirs can block this signaling cascade, resulting in reduction of viral pathogenicity and mortality. Adapted by permission from Macmillan Publishers Ltd: [Nature Communications] (66), copyright (2014). 


\section{CONCLUSION}

Recent studies have shown the importance of miRNAs in Enterovirus-related pathogenesis and hence, miRNAs provide an opportunity for the design of novel therapeutic and preventive strategies against viral infections [71]. It is interesting to note that a patent covering miRNA-based antiviral agents such as Miravirsen has been filed (WO 2013068348 A1) is currently undergoing clinical trial phase 3 (NCT 01200420). This miRNA antiviral agent elicits a prolonged dose-dependent decrease in in HCV RNA levels without evidence of viral resistance [72]. As there is currently no FDA-approved antiviral for EV-A71, antivirals that target host miRNA could be designed to prevent up-regulation of miRNAs that enhance virulence of EV-A71. In addition, the miRNA-based vaccine provides an interesting mechanism for controlling viral replication in specific tissues and yet it can still elicit a strong immunological response. There have been patents filed on miRNA-based vaccines such as the LAV against Influenza Virus (EP 2403527 A2) and Herpes Virus (EP 2303334 A1). If a miRNA-based vaccine or antiviral candidate for EV-A71 is developed, evaluation of cross-genotype protection, toxicity, pharmacokinetics and drug delivery studies will need to be conducted.

\section{CONFLICT OF INTEREST}

The authors declare no conflicts of interest.

\section{ACKNOWLEDGEMENTS}

We are grateful and acknowledge the support of Sunway University Internal Grants INT-VCO-0214-01 and INTVCO-0114-05, INT-RRO-2014-017 and the Fundamental Research Grant Scheme (FRGS) (FRGS/2/2014/ST03/SYUC//1) from the Malaysian Ministry of Education and Sunway Vice-Chancellor Research Fellowship to Isabel Yee.

\section{REFERENCES}

1. Ooi MH, Wong SC, Lewthwaite P, Cardosa MJ, Solomon T. Clinical features, diagnosis, and management of enterovirus 71. Lancet Neurol 2010; 9(11): 1097-105.

2. Schmidt N, Lennette E, Ho H. An apparently new enterovirus isolated from patients with disease of the central nervous system. J Infect Dis 1974; 129: 304 - 9.

3. Phuektes P, Chua BH, Sanders S, Bek EJ, Kok CC, McMinn PC. Mapping genetic determinants of the cell-culture growth phenotype of enterovirus 71. J Gen Virol 2011; 92(6): 1380-90.

4. Bible J, Pantelidis P, Chan P, Tong C. Genetic evolution of enterovirus 71: epidemiological and pathological implications. Rev Med Virol 2007; 17: 371 - 9.

5. Xing W, Liao Q, Viboud C, Zhang J, Sun J, Wu JT, et al. Hand, foot, and mouth disease in China, 2008-12: an epidemiological study. Lancet Infect Dis 2014; 14(4): 308-18.

6. Chong P, Liu CC, Chow YH, Chou AH, Klein M. Review of Enterovirus 71 Vaccines Clin Infect Dis. 2015; 60(5): 797-803.

7. Zhu FC, Meng FY, Li JX, Li XL, Mao QY, Tao H, et al. Efficacy, safety, and immunology of an inactivated alum-adjuvant enterovirus 71 vaccine in children in China: a multicentre, randomised, double-blind, placebo-controlled, phase 3 trial. The Lancet 2013; 381(9882): 2024-32.

8. Chang LY, Hsiung CA, Lu CY, Lin TY, Huang FY, Lai YH, et al. Status of cellular rather than humoral immunity is correlated with clinical outcome of enterovirus 71. Pediatr Res 2006; 60(4): 466-71.

9. Arita M, Nagata N, Iwata N, Ami Y, Suzaki Y, Mizuta K, et al. An Attenuated Strain of Enterovirus 71 Belonging to Genotype A Showed a Broad Spectrum of Antigenicity with Attenuated Neurovirulence in Cynomolgus Monkeys. J Virol 2007; 81(17): 9386-95. 
10. Pasquinelli AE, Reinhart BJ, Slack F, Martindale MQ, Kuroda MI, Maller B, et al. Conservation of the sequence and temporal expression of let-7 heterochronic regulatory RNA. Nature 2000; 408(6808): 86-9.

11. Bartel DP. MicroRNAs: Genomics, Biogenesis, Mechanism, and Function. Cell 2004; 116(2): $281-97$.

12. Skalsky RL, Cullen BR. Viruses, microRNAs, and host interactions. Annu Rev Microbiol 2010; 64:

$123-41$.

13. Lauring AS, Jones JO, Andino R. Rationalizing the development of live attenuated virus vaccines. Nat Biotechnol 2010; 28(6): 573-9.

14. tenOever BR. RNA viruses and the host microRNA machinery. Nat Rev Micro 2013; 11(3): 169-80.

15. Bartel DP. MicroRNAs: Target Recognition and Regulatory Functions. Cell 2009; 136(2): 215-33.

16. Chiang HR, Schoenfeld LW, Ruby JG, Auyeung VC, Spies N, Baek D, et al. Mammalian microRNAs: experimental evaluation of novel and previously annotated genes. Genes Dev 2010; 24(10): 992-1009.

17. Ghosh Z, Mallick B, Chakrabarti J. Cellular versus viral microRNAs in host-virus interaction. Nucleic Acids Res 2009; 37(4): 1035-48.

18. Mack GS. MicroRNA gets down to business. Nat Biotech 2007; 25(6): 631-8.

19. Nathans R, Chu CY, Serquina AK, Lu CC, Cao H, Rana TM. Cellular MicroRNA and P Bodies

Modulate Host-HIV-1 Interactions. Mol Cell 2009; 34(6): 696-709.

20. Scaria V, Hariharan M, Maiti S, Pillai B, Brahmachari SK. Host-virus interaction: a new role for microRNAs. Retrovirology 2006; 3: 68-76.

21. Archambaud C, Sismeiro O, Toedling J, Soubigou G, Bécavin C, Lechat P, et al. The intestinal microbiota interferes with the microRNA response upon oral listeria infection. mBio 2013; 4(6): e00707-13.

22. Cui L, Guo X, Qi Y, Qi X, Ge Y, Shi Z, et al. Identification of microRNAs involved in the host response to enterovirus 71 infection by a deep sequencing approach. J Biomed Biotechnol 2010; 2010: 425939.

23. Brown BD, Naldini L. Exploiting and antagonizing microRNA regulation for therapeutic and experimental applications. Nat Rev Genet 2009; 10(8): 578-85.

24. Brown BD, Venneri MA, Zingale A, Sergi LS, Naldini L. Endogenous microRNA regulation suppresses transgene expression in hematopoietic lineages and enables stable gene transfer. Nat Med 2006; 12(5): 585-91.

25. Kelly EJ, Hadac EM, Greiner S, Russell SJ. Engineering microRNA responsiveness to decrease virus pathogenicity. Nat Med 2008; 14(11): 1278-83.

26. Kelly EJ, Hadac EM, Cullen BR, Russell SJ. MicroRNA antagonism of the picornaviral life cycle: Alternative mechanisms of interference. PLoS Pathog 2010; 6(3): 1-11.

27. Barnes D, Kunitomi M, Vignuzzi M, Saksela K, Andino R. Harnessing endogenous miRNAs to control virus tissue tropism as a strategy for developing attenuated virus vaccines. Cell Host Microbe 2008; 4(3): 239248.

28. Cawood R, Chen HH, Carroll F, Bazan-Peregrino M, van Rooijen N, Seymour LW. Use of tissuespecific microRNA to control pathology of wild-type adenovirus without attenuation of its ability to kill cancer cells. PLoS Pathog 2009; 5(5): 1-10.

29. Perez JT, Pham AM, Lorini MH, Chua MA, Steel J, tenOever BR. MicroRNA-mediated speciesspecific attenuation of influenza A virus. Nat Biotech 2009; 27(6): 572-6.

30. Lee TC, Lin YL, Liao JT, Su CM, Lin CC, Lin WP, et al. Utilizing liver-specific microRNA-122 to modulate replication of dengue virus replicon. Biochem Biophys Res Commun 2010; 396(3): 596-601.

31. Vignuzzi M, Wendt E, Andino R. Engineering attenuated virus vaccines by controlling replication fidelity. Nat Med 2008; 14(2): 154-61.

32. Henke JI, Goergen D, Zheng J, Song Y, Schüttler CG, Fehr C, et al. microRNA-122 stimulates translation of hepatitis C virus RNA. EMBO J 2008; 27(24): 3300-10.

33. Li Y, Masaki T, Yamane D, McGivern DR, Lemon SM. Competing and noncompeting activities of miR-122 and the 5' exonuclease Xrn 1 in regulation of hepatitis C virus replication. PNAS 2013; 110(5): 1881-6. 34. Klotman ME, Chang TL. Defensins in innate antiviral immunity. Nat Rev Immunol 2006; 6(6): 447456.

35. Wilson SS, Wiens ME, Smith JG. Antiviral mechanisms of human defensins. J Mol Biol 2013; 425(24): 1-26.

36. Kawai T, Akira S. Innate immune recognition of viral infection. Nat Immunol 2006;7(2):131-7.

37. Thompson MR, Kaminski JJ, Kurt-Jones EA, Fitzgerald KA. Pattern recognition receptors and the innate immune response to viral infection. Viruses 2011; 3(6): 920.

38. Nguyen KB, Watford WT, Salomon R, Hofmann SR, Pien GC, Morinobu A, et al. Critical Role for STAT4 Activation by Type 1 Interferons in the Interferon- $\gamma$ Response to Viral Infection. Science 2002; 297(5589): 2063-6.

39. Liu ML, Lee YP, Wang YF, Lei HY, Liu CC, Wang SM, et al. Type I interferons protect mice against enterovirus 71 infection. J Gen Virol 2005; 86(12): 3263-9. 
40. Lu J, Yi L, Zhao J, Yu J, Chen Y, Lin MC, et al. Enterovirus 71 Disrupts Interferon Signaling by Reducing the Level of Interferon Receptor 1. J Virol 2012; 86(7): 3767-76.

41. Li Y, Xie J, Xu X, Wang J, Ao F, Wan Y, et al. MicroRNA-548 down-regulates host antiviral response via direct targeting of IFN- $\lambda 1$. Protein Cell 2012; 4(2): 130-41.

42. Lui YLE, Tan TL, Woo WH, Timms P, Hafner LM, Tan KH, et al. Enterovirus71 (EV71) Utilise Host microRNAs to Mediate Host Immune System Enhancing Survival during Infection. PLoS ONE 2014; 9(7): 1-

12.

43. Ho BC, Yu SL, Chen JJW, Chang SY, Yan BS, Hong QS, et al. Enterovirus-Induced miR-141 Contributes to Shutoff of Host Protein Translation by Targeting the Translation Initiation Factor eIF4E. Cell Host Microbe 2011; 9(1): 58-69.

44. Zheng Z, Ke X, Wang M, He S, Li Q, Zheng C, et al. Human MicroRNA hsa-miR-296-5p Suppresses Enterovirus 71 Replication by Targeting the Viral Genome. J Virol 2013;87(10):5645-56.

45. Wen B, Dai H, Yang Y, Zhuang Y, Sheng R. MicroRNA-23b Inhibits Enterovirus 71 Replication through Downregulation of EV71 VPl Protein. Intervirology 2013; 56(3): 195-200.

46. Du X, Wang H, Xu F, Huang Y, Liu Z, Liu TE. Enterovirus 71 induces apoptosis of SH-SY5Y human neuroblastoma cells through stimulation of endogenous microRNA let-7b expression. Mol Med Rep 2015; 12(1): 953-9.

47. Xu LJ, Jiang T, Zhao W, Han JF, Liu J, Deng YQ, et al. Parallel mRNA and microRNA profiling of HEV71-infected human neuroblastoma cells reveal the up-regulation of miR-1246 in association with DLG3 repression. PLoS One 2014; 9(4): 1-9.

48. Soifer HS, Rossi JJ, Saetrom P. MicroRNAs in Disease and Potential Therapeutic Applications. Mol Ther 2007; 15(12): 2070-9.

49. Wurdinger T, Costa FF. Molecular therapy in the microRNA era. Pharmacogenomics J 2006; 7(5): 297-304.

50. Davis S, Lollo B, Freier S, Esau C. Improved targeting of miRNA with antisense oligonucleotides. Nucleic Acids Res 2006; 34(8): 2294-304.

51. Hutvágner G, Simard MJ, Mello CC, Zamore PD. Sequence-Specific Inhibition of Small RNA Function. PLoS Biol 2004; 2(4): 465-75.

52. Meister G, Landthaler M, Dorsett Y, Tuschl T. Sequence-specific inhibition of microRNA- and siRNA-induced RNA silencing. RNA 2004; 10(3): 544-50.

53. Boutla A, Delidakis C, Tabler M. Developmental defects by antisense-mediated inactivation of microRNAs 2 and 13 in Drosophila and the identification of putative target genes. Nucleic Acids Res 2003; 31(17): 4973-80.

54. Ørom UA, Kauppinen S, Lund AH. LNA-modified oligonucleotides mediate specific inhibition of microRNA function. Gene 2006; 372: 137-41.

55. Leaman D, Chen PY, Fak J, Yalcin A, Pearce M, Unnerstall U, et al. Antisense-Mediated Depletion Reveals Essential and Specific Functions of MicroRNAs in Drosophila Development. Cell 2005; 121(7): 1097108.

56. Wiemer EAC. The role of microRNAs in cancer: No small matter. Eur J Cancer 2007; 43(10): 1529-

44.

57. Calin GA, Dumitru CD, Shimizu M, Bichi R, Zupo S, Noch E, et al. Frequent deletions and downregulation of micro- RNA genes miR15 and miR16 at 13q14 in chronic lymphocytic leukemia. PNAS 2002; 99(24): 15524-9.

58. Calin GA, Liu C-G, Sevignani C, Ferracin M, Felli N, Dumitru CD, et al. MicroRNA profiling reveals distinct signatures in B cell chronic lymphocytic leukemias. PNAS 2004; 101(32): 11755-60.

59. Yang M, Mattes J. Discovery, biology and therapeutic potential of RNA interference, microRNA and antagomirs. Pharmacol Ther 2008; 117(1): 94-104.

60. Bijsterbosch MK, Rump ET, Vrueh RLAD, Dorland R, Veghel Rv, Tivel KL, et al. Modulation of plasma protein binding and in vivo liver cell uptake of phosphorothioate oligodeoxynucleotides by cholesterol conjugation. Nucleic Acids Res 2000; 28(14): 2717-25.

61. van Rooij E, Purcell AL, Levin AA. Developing MicroRNA Therapeutics. Circ Res 2012; 110(3): 496507.

62. Weiler J, Hunziker J, Hall J. Anti-miRNA oligonucleotides (AMOs): ammunition to target miRNAs implicated in human disease? Gene Ther 2005; 13(6): 496-502.

63. Liu Z, Sall A, Yang D. MicroRNA: An emerging therapeutic target and intervention tool. Int J Mol Sci 2008; 9(6): 978-99.

64. Grimson A, Farh KKH, Johnston WK, Garrett-Engele P, Lim LP, Bartel DP. MicroRNA Targeting Specificity in Mammals: Determinants Beyond Seed Pairing. Mol Cell 2007; 27(1): 91-105.

65. Lu J, Yi L, Zhao J, Yu J, Chen Y, Lin MC, et al. Enterovirus 71 Disrupts Interferon Signaling by reducing the Interferon Receptor 1. J Virol 2012; 86: 3767- 3776. 
66. Ho BC, Yu IS, Lu LF, Rudensky A, Chen HY, Tsai CW, et al. Inhibition of miR-146a prevents enterovirus-induced death by restoring the production of type I interferon. Nat Commun 2014; 5: 1-12.

67. Farooqi AA, Fayyaz S, Shatynska-Mytsyk I, Javed Z, Jabeen S, Yaylim I, et al. Is miR-34a a Wellequipped Swordsman to Conquer Temple of Molecular Oncology. Chem Biol Drug Des 2016; 87: 321-34.

68. Gebert LFR, Rebhan MAE, Crivelli SEM, Denzler R, Stoffel M, Hall J. Miravirsen (SPC3649) can inhibit the biogenesis of miR-122. Nucleic Acids Res 2014; 42(1): 609-21.

69. Tan CW, Chan YF, Quah YW, Poh CL. Inhibition of enterovirus 71 infection by antisense octaguanidinium dendrimer-conjugated morpholino oligomers. Antiviral Res 2014; 107: 35-41.

70. Kloosterman WP, Lagendijk AK, Ketting RF, Moulton JD, Plasterk RHA. Targeted Inhibition of miRNA Maturation with Morpholinos Reveals a Role for miR-375 in Pancreatic Islet Development. PLoS Biol 2007; 5(8): 1738-1749.

71. Ho BC, Yang PC, Yu SL. MicroRNA and pathogenesis of enterovirus infection. Viruses. 2016; 8(1): 11-25.

72. Janssen HLA, Reesink HW, Lawitz EJ, Zeuzem S, Rodriguez-Torres M, Patel K et al. Treatment of HCV infection by targeting microRNA. New England Journal of Medicine. 2013; 368(18): 1685-1694. 